\title{
Handgrip Strength Is an Independent Predictor of Cardiovascular Outcomes in Diabetes Mellitus
}

\author{
Yoshinobu Morikawa, ${ }^{1}$ MD, Rika Kawakami, ${ }^{2}$ MD, Manabu Horii, ${ }^{1}$ MD, Yuta Yamamoto, ${ }^{1}$ MD, \\ Matahiro Yabuta, ${ }^{3} \mathrm{MD}$ and Yoshihiko Saito, ${ }^{2} \mathrm{MD}$
}

\begin{abstract}
Summary
Diabetes mellitus (DM) is associated with an increased incidence of cardiovascular events and an elevated prevalence of sarcopenia. However, the relationship between cardiovascular events and sarcopenia in patients with DM remains unclear. This study examined this relationship and investigated the predictors of cardiovascular events in this population.

This study enrolled 161 patients with DM and no history of cardiovascular diseases who were admitted to our hospital for the treatment of DM between September 2012 and December 2015. Patients were divided into sarcopenia and non-sarcopenia groups, and were followed until March 2019. The primary endpoint was major adverse cardiovascular events (MACE).

The mean age was $65.9 \pm 1.8$ years old and the mean follow-up period was $4.1 \pm 0.8$ years. The log-rank test indicated that MACE differed significantly between the two groups $(P<0.0001)$. Multivariate Cox hazard analysis identified the cardio-ankle vascular index (CAVI) and handgrip strength as independent predictors of MACE (hazard ratio $[\mathrm{HR}]=1.18, P=0.039$; and $\mathrm{HR}=0.70, P=0.016$, respectively).

Handgrip strength is an indicator of sarcopenia in diabetic patients, and together with CAVI it was independently associated with the incidence of MACE. This suggests that the handgrip strength test might be useful in the management of patients with DM at high risk of cardiovascular outcomes.
\end{abstract}

(Int Heart J 2021; 62: 50-56)

Key words: Skeletal muscle strength, Sarcopenia, Cardiovascular disease

I n 1989, Rosenberg proposed the term sarcopenia, which refers to the involuntary loss of muscle mass associated with aging, ${ }^{1-4)}$ where this progressive decline in skeletal muscle mass is a serious adverse change associated with human aging. The condition has important implications regarding health outcomes and affects physical function, as manifested by high rates of disability in the elderly. ${ }^{5}$

Compared with non-diabetic patients, those with diabetes mellitus (DM) have a higher incidence of arteriosclerotic diseases such as coronary disease and stroke. A meta-analysis of prospective cohort studies worldwide showed that, compared to non-diabetic patients, male patients with DM had about a two-fold higher risk of coronary disease and female patients with DM had a three- to four-fold higher risk. ${ }^{6}$ )

While loss of skeletal muscle strength and quality is accelerated in patients with DM and insulin resistance, ${ }^{7,8}$ it is also known that the prevalence of sarcopenia is higher in patients with DM than in those without. Furthermore, some recent studies showed that sarcopenia was closely associated with coronary artery disease and heart failure. $^{9-11)}$
However, the relationship between cardiovascular events and sarcopenia in patients with DM has not yet been fully investigated. Furthermore, the predictors of cardiovascular events, including sarcopenia, have not been sufficiently studied. In this study, we examined the relationships between sarcopenia and all-cause mortality, cardiovascular events, and hospitalization for heart failure in patients with DM.

\section{Methods}

Study subjects: The participants in this study were 189 patients admitted to our hospital for treatment of DM between September 2012 and December 2015. Diabetes was defined as current treatment with oral hypoglycemic agents or insulin or a fasting glucose level $\geq 126 \mathrm{mg} / \mathrm{dL}$. The participants were divided into two groups based on sarcopenia status. We excluded patients with recent acute coronary syndrome $(n=1)$, those with angiographically confirmed coronary artery disease who were undergoing percutaneous coronary angioplasty $(n=6)$, and those with known malignant disease $(n=3)$. During hospitalization, a treadmill test was performed to evaluate ischemic heart

From the ${ }^{1}$ Department of Cardiovascular Medicine, Nara City Hospital, Nara, Japan, ${ }^{2}$ Department of Cardiovascular Medicine, Nara Medical University, Nara, Japan and ${ }^{3}$ Department of Diabetic Medicine, Nara City Hospital, Nara, Japan.

Address for correspondence: Yoshinobu Morikawa, MD, Department of Cardiovascular Medicine, Nara City Hospital, 1-50-1 Higashi-Kidera Nara-City, Nara 630-8305, Japan. E-mail: ymorikawa@ heart.so-net.jp

Received for publication October 7, 2020. Revised and accepted November 18, 2020.

doi: 10.1536/ihj.20-677

All rights reserved by the International Heart Journal Association. 


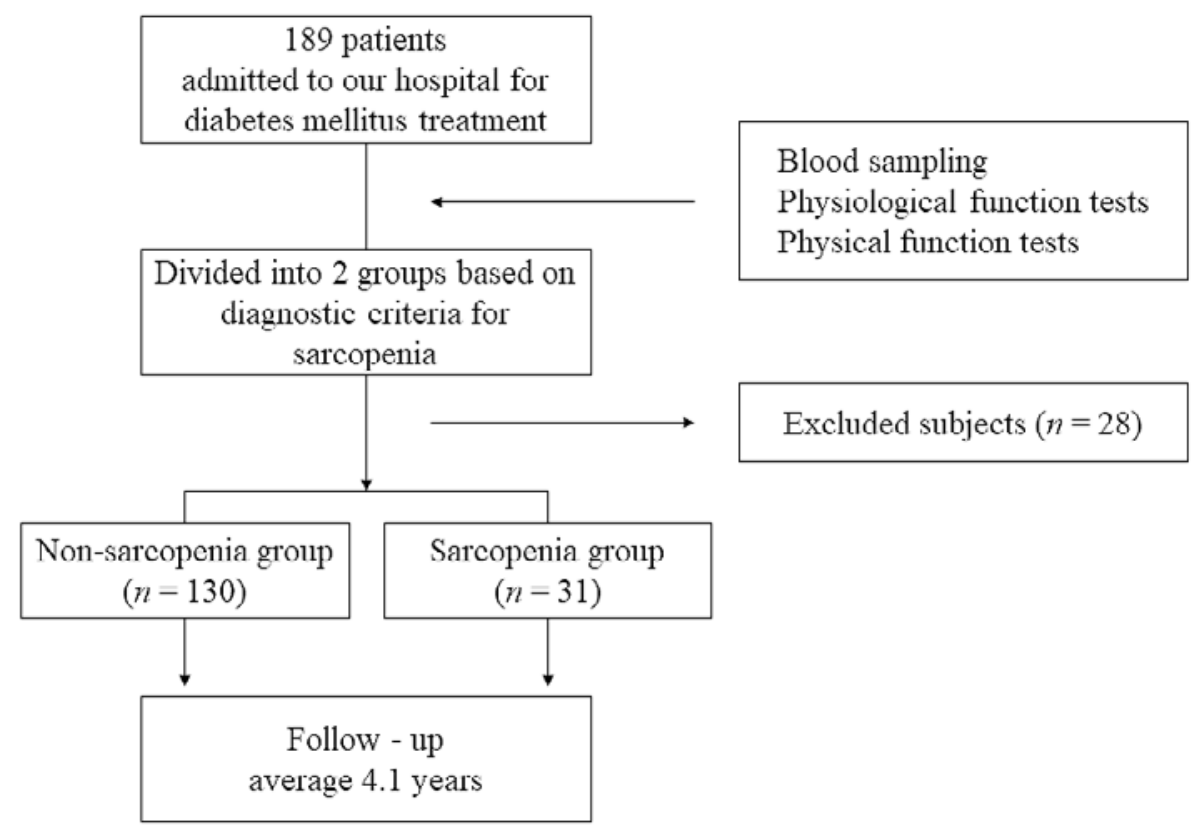

Figure 1. The participants in this study were 189 patients admitted to our hospital between September 2012 and December 2015 for the purpose of treating diabetes mellitus. The participants were divided into two groups based on sarcopenia status. We excluded 28 patients for several reasons. After discharge, patients were followed prospectively in an ambulatory care setting until March 2019, with a mean follow-up period of $4.1 \pm 0.8$ years.

disease, and those who did not reach the target heart rate $(n=5)$ or who demonstrated ischemia $(n=3)$ were excluded. Ten patients lacked follow-up data, either due to disability or institutionalization $(n=5)$ or because they were lost to follow-up $(n=5)$. After discharge from our hospital, patients were followed up prospectively in an ambulatory care setting until March 2019, with a mean follow-up period of $4.1 \pm 0.8$ years (Figure 1).

This study was conducted in accordance with the Declaration of Helsinki and was approved by the Ethics Committee of Nara City Hospital (approval number 36). Data compilation was performed in a manner intended to prevent identification of patients' personal information. In addition, the study analyzed the results of evaluations performed during a series of hospitalizations for diabetes education. The study was fully explained to all participants, and analysis was performed only after written consent was obtained.

Primary and secondary endpoints: The primary endpoint of this study was a composite of major adverse cardiovascular events (MACE: cardiovascular death, nonfatal myocardial infarction $[\mathrm{MI}]$, unstable angina, newly developed effort angina, heart failure, and stroke). Secondary endpoints comprised each of the separate cardiovascular events included in MACE.

Definition of sarcopenia: This study adopted the diagnostic criteria in the consensus report of the Asian Working Group for Sarcopenia." The presence of low muscle mass and poor strength was used to diagnose sarcopenia. Patients with a walking speed higher than $0.8 \mathrm{~m} / \mathrm{second}$ were assigned to the non-sarcopenia group. We used the appendicular skeletal muscle mass index to measure mus- cle mass and the handgrip strength test to examine muscle strength. Appendicular skeletal muscle mass (ASM) was calculated by summing the muscle mass in the arms and legs, assuming that all tissues that were not fat or bone were skeletal muscle. The appendicular skeletal muscle mass index (SMI) was defined as ASM/height ${ }^{2}\left(\mathrm{~kg} / \mathrm{m}^{2}\right) .{ }^{12)}$ An ASM reference value of $7.0 \mathrm{~kg} / \mathrm{m}^{2}$ was used to diagnose sarcopenia in men and $5.7 \mathrm{~kg} / \mathrm{m}^{2}$ was used in women. The cut-off points for handgrip strength were 26 $\mathrm{kg}$ in men and $18 \mathrm{~kg}$ in women. Based on the diagnostic criteria mentioned above, we divided patients into sarcopenia and non-sarcopenia groups.

Anthropometric measurement: In this study, lean mass of the upper and lower extremities and total body lean mass were assessed using bioelectrical impedance analysis (BIA) conducted with an InBody 520 body composition analyzer (Model D; Biospace, Seoul, Korea). BIA measurements were taken at least two hours after breakfast and with an empty bladder.

Strength assessment: Isokinetic knee extensor strength was measured with a dynamometer (model $\mu$ Tas; Anima, Tokyo, Japan) with the participant in a sitting position with the knee at 90 degrees of flexion. Both left and right sides were measured unless one side was injured or if exercise was contraindicated. Handgrip strength was measured with a hand dynamometer (Model Grip D; Takei, Japan) with the participant in the sitting position with the elbow flexed at 90 degrees. For both knee extensor and handgrip strengths, the strength was defined as the average of the right and left sides.

Evaluation of echocardiography, flow-mediated dilatation (FMD) and the cardio-ankle vascular index 
Table I. Comparisons of Laboratory and Physiological Data in Sarcopenia and Non-Sarcopenia Groups $(n=161)$

\begin{tabular}{|c|c|c|c|}
\hline Variables & $\begin{array}{c}\text { Sarcopenia } \\
\quad(n=31)\end{array}$ & $\begin{array}{l}\text { Non-Sarcopenia } \\
\quad(n=130)\end{array}$ & $P$ value \\
\hline Age (years) & $67.1 \pm 2.4$ & $65.2 \pm 0.8$ & 0.327 \\
\hline Gender (female/ male) & $14 / 17$ & $69 / 61$ & 0.428 \\
\hline Duration of diabetes (years) & $13.3 \pm 1.8$ & $12.8 \pm 1.0$ & 0.458 \\
\hline Body mass index $\left(\mathrm{kg} / \mathrm{m}^{2}\right)$ & $21.1 \pm 0.9$ & $25.8 \pm 0.5$ & $<0.0001$ \\
\hline HDL cholesterol (mg/dL) & $52.1 \pm 2.6$ & $52.5 \pm 1.2$ & 0.903 \\
\hline LDL cholesterol (mg/dL) & $112.9 \pm 6.4$ & $123.1 \pm 3.5$ & 0.100 \\
\hline Triglyceride (mg/dL) & $133.7 \pm 22.1$ & $153.1 \pm 10.7$ & 0.214 \\
\hline $\mathrm{HbAlc}(\%)$ & $10.5 \pm 0.4$ & $10.2 \pm 0.3$ & 0.299 \\
\hline C-reactive protein $(\mathrm{mg} / \mathrm{dL})$ & $0.24 \pm 0.08$ & $0.12 \pm 0.06$ & 0.125 \\
\hline Serum creatinine $(\mathrm{mg} / \mathrm{dL})$ & $0.75 \pm 0.05$ & $0.73 \pm 0.01$ & 0.751 \\
\hline Albumin $(\mathrm{g} / \mathrm{dL})$ & $3.73 \pm 0.08$ & $3.9 \pm 0.05$ & 0.572 \\
\hline Albuminuria $(\mathrm{mg} / \mathrm{gCr})$ & $102.1 \pm 19.8$ & $43.1 \pm 7.1$ & 0.005 \\
\hline NT-proBNP (pg/mL) & $96.8 \pm 20.1$ & $65.3 \pm 8.8$ & 0.318 \\
\hline FMD $(\%)$ & $4.0 \pm 0.6$ & $6.3 \pm 0.2$ & 0.0002 \\
\hline CVRR (\%) & $2.26 \pm 0.24$ & $2.46 \pm 0.12$ & 0.493 \\
\hline LVEF (\%) & $71.2 \pm 1.9$ & $69.9 \pm 0.8$ & 0.391 \\
\hline $\mathrm{E} / \mathrm{E}$ & $13.8 \pm 0.6$ & $11.5 \pm 0.3$ & 0.0002 \\
\hline CAVI (m/second) & $9.95 \pm 0.24$ & $8.51 \pm 0.11$ & $<0.0001$ \\
\hline \multicolumn{4}{|l|}{ Nerve conduction } \\
\hline Elbow & $50.0 \pm 0.9$ & $50.7 \pm 0.5$ & 0.443 \\
\hline Popliteal & $41.3 \pm 0.7$ & $41.0 \pm 0.4$ & 0.612 \\
\hline Handgrip strength (kg) & $23.1 \pm 1.6$ & $30.5 \pm 1.0$ & 0.0007 \\
\hline $\operatorname{SMI}\left(\mathrm{kg} / \mathrm{m}^{2}\right)$ & $6.6 \pm 0.3$ & $8.5 \pm 0.1$ & $<0.0001$ \\
\hline Hypertension $n(\%)$ & $12(38.7)$ & $47(36.2)$ & 0.791 \\
\hline Dyslipidemia $n(\%)$ & $4(12.9)$ & $35(26.9)$ & 0.102 \\
\hline Current smoker $n(\%)$ & $6(19.3)$ & $17(13.1)$ & 0.369 \\
\hline \multicolumn{4}{|l|}{ Medication $n(\%)$} \\
\hline Biguanide & $2(6.5)$ & $32(23.1)$ & 0.026 \\
\hline Thiazolidine & $3(9.7)$ & $8(6.2)$ & 0.485 \\
\hline DPP-4 inhibitor & $7(22.6)$ & $37(28.4)$ & 0.509 \\
\hline Sulfonylurea & $5(16.1)$ & $26(20.0)$ & 0.623 \\
\hline$\alpha$-glucosidase inhibitor & $3(9.7)$ & $18(13.8)$ & 0.536 \\
\hline SLGT2 inhibitor & $2(6.5)$ & $9(6.9)$ & 0.926 \\
\hline Insulin & $9(29.0)$ & $53(40.8)$ & 0.228 \\
\hline Stain & $4(12.9)$ & $19(14.6)$ & 0.807 \\
\hline ACEI or ARB & $8(25.8)$ & $32(24.6)$ & 0.890 \\
\hline Aspirin & $2(6.5)$ & $7(5.4)$ & 0.816 \\
\hline
\end{tabular}

Data are expressed as mean \pm SE or as numbers. Comparisons between the two groups were performed using the Wilcoxon signed-rank test. HDL indicates high-density lipoprotein; LDL, low-density lipoprotein; FMD, flow-mediated dilatation; CVRR, coefficient variation of R-R intervals; CAVI, cardio-ankle vascular index; LVEF, left ventricular ejection fraction; SMI, skeletal muscle mass index; DPP-4, dipeptidyl-peptidase 4; and SLGT2, sodium-glucose cotransporter 2.

(CAVI): We measured left ventricular diameter, left ventricular ejection fraction, E-wave, E'-wave (mean of septal and lateral wall), and $\mathrm{E} / \mathrm{E}$ ' ratio as echocardiographic parameters.

The analysis of FMD was performed by strictly following the protocols of Corretti, et al. ${ }^{13)}$ and Thijssen, et al. ${ }^{14)}$ Since various factors affect FMD, we standardized temperature, food, drugs, and sympathetic stimulation in the experimental setup. Patients fasted and avoided exercise, caffeine, alcohol, drugs, stimulants, and medications for 12 hours before the FMD examination. Endotheliumdependent dilatation of the brachial artery was measured using ultrasound (UNEXEF; UNEX Corporation, Nagoya, Japan). CAVI was measured in a supine position using a Vasara VS-1000 (Fukuda Denshi, Tokyo, Japan) in accor- dance with the protocol described in a previous study. ${ }^{15)}$ The average of the left and right CAVI values was used for analysis.

Laboratory measurement: Fasting blood samples were taken in the morning after hospital admission. Fasting glucose, total cholesterol, high-density lipoprotein cholesterol, low-density lipoprotein cholesterol, triglycerides, Creactive protein, and $\mathrm{N}$-terminal pro-brain natriuretic peptide (NT-proBNP) levels were measured using an autoanalyzer (Automatic Analyzer 7600; Hitachi, Tokyo, Japan). We used high-performance liquid chromatography to measure urinary albumin. Both urinary albumin and creatinine were measured using early morning spot urine samples.

Statistical analysis: Statistical analysis was performed us- 
$P<0.0001$

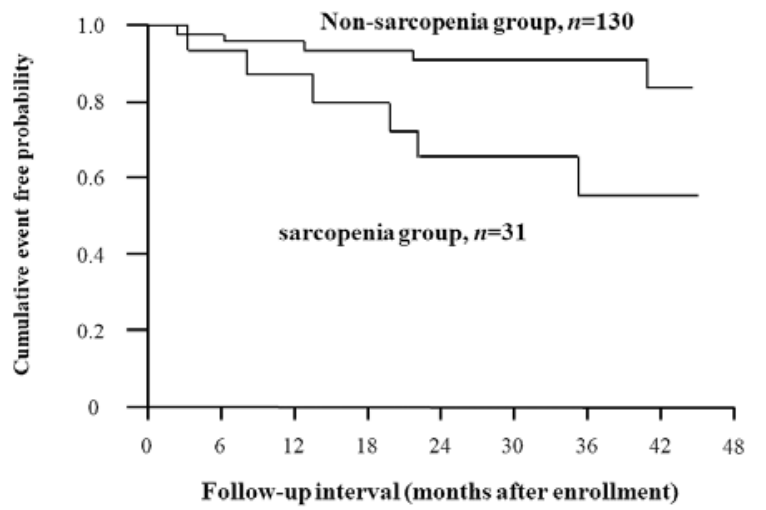

Figure 2. Primary endpoint: composite of all events. The log-rank test indicates a significant difference between the two groups in the incidence of MACE $(P<0.0001)$. ing JMP software, version 10.0.2 (SAS Institute, Cary, $\mathrm{NC}$, USA). Numerical data were expressed as means $( \pm$ standard error) or discrete values (numbers or percentages). The Wilcoxon signed-rank test was used to compare the laboratory, physiological, and physical function test results between the two groups. Pearson's chi-square test was performed on the nominal scales. The log-rank test was used to compare the incidence of events between two groups. Cox hazard analysis was used to evaluate MACE in univariable and multivariable analyses. Covariates with a significance of $P<0.05$ or below in univariate analyses were included in the multivariate stepwise analysis. All $P$-values were 2 -tailed, and $P<0.05$ was considered statistically significant. Receiver operating characteristic (ROC) analysis and the area under curve (AUC) were used to identify the sensitivity and specificity of handgrip strength cut-off points for the detection of MACE. The optimal cut-off values were defined as the point at which the value of "sensitivity + specificity-1" was maximum (Youden index).

Table II. Log-Rank Test Comparing the Incidence of Events between Two Groups ( $n=161)$

\begin{tabular}{|c|c|c|c|c|c|c|}
\hline \multirow{2}{*}{ Outcomes } & \multicolumn{2}{|c|}{$\begin{array}{l}\text { Sarcopenia } \\
\quad(n=31)\end{array}$} & \multicolumn{2}{|c|}{$\begin{array}{l}\text { Non-Sarcopenia } \\
\quad(n=130)\end{array}$} & \multirow[t]{2}{*}{$\begin{array}{l}\text { Hazard Ratio } \\
(95 \% \mathrm{CI})\end{array}$} & \multirow[t]{2}{*}{$P$ value } \\
\hline & No. $(\%)$ & $\begin{array}{l}\text { No. per } 100 \\
\text { person-year }\end{array}$ & No. $(\%)$ & $\begin{array}{l}\text { No. per } 100 \\
\text { person-year }\end{array}$ & & \\
\hline Primary end point: all events & $7(22.6)$ & 5.54 & $6(4.6)$ & 1.13 & $4.71(2.10-6.72)$ & $<0.0001$ \\
\hline Cardiovascular death & $0(0)$ & 0 & $0(0)$ & 0 & - & \\
\hline Nonfatal myocardial infarction & $1(3.2)$ & 0.68 & $0(0)$ & 0 & - & \\
\hline Effort angina & $3(9.6)$ & 2.37 & $2(1.5)$ & 0.38 & $6.15(3.49-8.26)$ & 0.027 \\
\hline Heart failure & $1(3.2)$ & 0.79 & $2(1.5)$ & 0.38 & $2.07(1.12-2.77)$ & 0.215 \\
\hline Stroke (ischemic and hemorrhagic) & $2(6.5)$ & 1.58 & $2(1.5)$ & 0.38 & $4.28(3.25-5.90)$ & 0.108 \\
\hline
\end{tabular}

Table III. Results of Univariate Cox Proportional Hazard Analysis of MACE During the Observation Period

\begin{tabular}{lcc}
\hline & \multicolumn{2}{c}{ Univariate analysis } \\
& HR $(95 \%$ CI $)$ & $P$ value \\
\hline Age $($ years $)$ & $1.00(0.98-1.03)$ & 0.507 \\
Gender (female/ male) & $1.01(0.45-2.30)$ & 0.975 \\
Duration of diabetes mellitus (years) & $1.00(0.97-1.02)$ & 0.702 \\
Body mass index $\left(\mathrm{kg} / \mathrm{m}^{2}\right)$ & $0.97(0.90-1.04)$ & 0.335 \\
Serum creatinine $(\mathrm{mg} / \mathrm{dL})$ & $1.60(0.61-3.81)$ & 0.328 \\
Albuminuria $(\mathrm{mg} / \mathrm{gCr})$ & $1.16(0.96-1.37)$ & 0.033 \\
NT-proBNP $(\mathrm{pg} / \mathrm{mL})$ & $1.00(0.99-1.01)$ & 0.547 \\
FMD $(\%)$ & $0.96(0.90-1.01)$ & 0.142 \\
LVEF $(\%)$ & $1.00(0.98-1.02)$ & 0.934 \\
E/E' & $1.35(0.84-1.85)$ & 0.012 \\
CAVI $(\mathrm{m} / \mathrm{second})$ & $1.49(0.88-2.11)$ & 0.001 \\
Handgrip strength $(\mathrm{kg})$ & $0.79(0.34-1.26)$ & 0.0002 \\
SMI $\left(\mathrm{kg} / \mathrm{m}^{2}\right)$ & $1.07(0.95-1.18)$ & 0.146 \\
Hypertension & $1.21(0.81-1.63)$ & 0.302 \\
Dyslipidemia & $1.18(0.73-1.87)$ & 0.486 \\
Current smoker & $1.13(0.65-2.05)$ & 0.662 \\
\hline
\end{tabular}

$\overline{\text { Independent variables }(P<0.05) \text { are listed. FMD indicates flow-mediated }}$ dilatation; CAVI, cardio-ankle vascular index; and SMI, skeletal muscle mass index. 
Table IV. Results of Multivariate Cox Proportional Hazard Analysis of MACE During the Observation Period

\begin{tabular}{lcc}
\hline & \multicolumn{2}{c}{ Multivariate analysis } \\
& HR $(95 \% \mathrm{CI})$ & $P$ value \\
\hline Albuminuria $(\mathrm{mg} / \mathrm{gCr})$ & $1.05(1.00-1.11)$ & 0.478 \\
E/E' & $1.09(0.97-1.13)$ & 0.102 \\
CAVI (m/second) & $1.18(0.99-1.21)$ & 0.039 \\
Handgrip strength $(\mathrm{kg})$ & $0.70(0.40-1.45)$ & 0.016 \\
\hline
\end{tabular}

Independent variables $(P<0.05)$ are listed. CAVI indicates cardio anklevascular index.

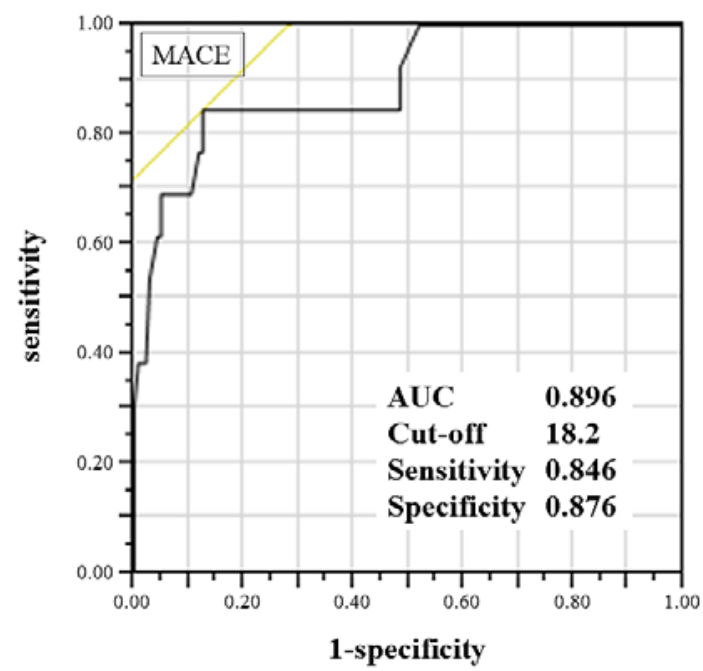

Figure 3. ROC curve of handgrip strength for MACE in this study.

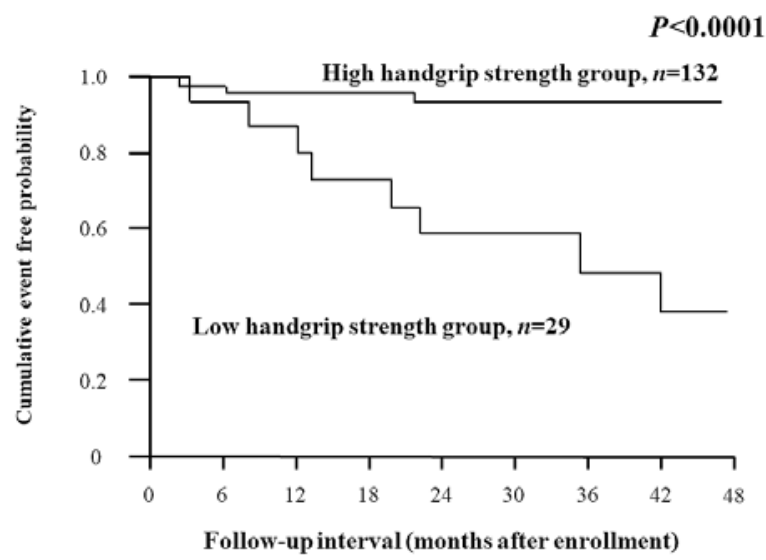

Figure 4. Primary endpoint: composite of all events. The log-rank test indicates a significant difference between the high and low handgrip strength groups in the incidence of MACE $(P<0.0001)$.

\section{Results}

Clinical characteristics: The data from 161 patients were available for the analysis of laboratory and physiological function test results. The baseline clinical characteristics of the 161 patients are shown in Table I. Patient age and the duration of DM did not differ between the sarcopenia and non-sarcopenia groups $(67.1 \pm 2.4$ versus $65.2 \pm 0.8$ years, $P=0.327 ; 13.2 \pm 1.7$ versus. $12.6 \pm 0.9$ years, $P=$ 0.451 , respectively). There were no significant differences in gender ratio between the two groups (female/male: 14/ 17 versus. $69 / 61, P=0.428$, respectively). Body mass index, FMD, SMI, and handgrip strength were significantly lower in the sarcopenia group than in the non-sarcopenia group $(21.1 \pm 0.9$ versus. $25.8 \% \pm 0.5 \%, P<0.0001 ; 4.0$ \pm 0.6 versus. $6.3 \% \pm 0.2 \%, P=0.0002 ; 6.6 \pm 0.3$ versus. $8.5 \% \pm 0.1 \%, P<0.0001 ; 23.1 \pm 1.6$ versus. $30.5 \pm 1.0$ $\mathrm{kg}, P=0.0007$, respectively). Albuminuria, E/E', and CAVI were significantly higher in the sarcopenia group than in the non-sarcopenia group $(102.1 \pm 19.8$ versus. $43.1 \pm 7.1 \mathrm{mg} / \mathrm{gCr}, P=0.005 ; 13.8 \pm 0.6$ versus. $11.5 \pm$ $0.3, P=0.0002 ; 9.95 \pm 0.2 .4$ versus $8.51 \pm 0.11 \mathrm{~m} / \mathrm{sec}-$ ond, $P<0.0001$, respectively). There were no significant differences between the sarcopenia and non-sarcopenia groups in the frequency of each drug used to treat diabetes, except for biguanides $(6.5 \%$ versus. $23.1 \%, P=$ 0.026 , respectively). The number of type 1 diabetic patients was three in the sarcopenia group and eight in the non-sarcopenia group, and there was no significant difference between two groups $(P=0.485)$.

The log-rank tests for primary and secondary endpoints: The log-rank test revealed a significant difference between the two groups in the incidence of MACE $(P<$ 0.0001) (Figure 2). In terms of secondary endpoints, the log-rank test showed that the two groups differed significantly in the incidence of effort angina $(P=0.027)$. On the other hand, there were no significant differences regarding hospitalization for heart failure $(P=0.215)$ or hospitalization for stroke $(P=0.108)$ (Table II).

Cox proportional analysis of MACE: Table III shows the results of univariate Cox proportional hazard analysis of MACE. Albuminuria, E/E', CAVI, and handgrip strength correlated significantly with MACE (hazard ratio $[\mathrm{HR}]=1.16, P=0.033$; HR $=1.35, P=0.012 ; \mathrm{HR}=$ $1.49, P=0.001 ; \mathrm{HR}=0.79, P=0.0002$, respectively). Table IV shows that the stepwise multivariate Cox hazard analysis identified CAVI and handgrip strength as independent predictive factors for MACE $(\mathrm{HR}=1.18, P=$ $0.039 ; \mathrm{HR}=0.70, P=0.016$, respectively).

ROC curve and the log-rank tests for MACE: Figure 3 shows the ROC curve for MACE in this study. This ROC curve revealed that $18.2 \mathrm{~kg}$ was the handgrip strength cutoff point for MACE (AUC: 0.896, Cut-off: 18.2, Sensitivity: 0.846, Specificity: 0.876). We divided the study into two groups with cut-off values of less than $18.2 \mathrm{~kg}$ (low handgrip strength) and $18.2 \mathrm{~kg}$ or more (high handgrip strength), and performed a log-rank test for MACE. The log-rank test indicates a significant difference between the low and high handgrip strength groups in the incidence of MACE $(P<0.0001)$ (Figure 4$)$.

\section{Discussion}

While Cox proportional hazard analysis of MACE identified CAVI value and handgrip strength as independent predictive factors, in this study the probability of MACE in the sarcopenia group was higher than that in the non-sarcopenia group. The main finding of the study was that handgrip strength was the most important and in- 
dependent predictor of MACE in patients with DM.

The relationship between sarcopenia and cardiovascular events was demonstrated in previous studies. ${ }^{16,17)}$ Among studies not focused on diabetes, while several showed that low handgrip strength was an effective predictor of cardiovascular events, ${ }^{18,19)}$ one suggested that handgrip strength was inversely associated with all-cause mortality and CVD. ${ }^{20)}$ While the association between sarcopenia and cardiovascular events in diabetic patients has not yet been fully investigated, a study of patients with DM, including those with previous cardiovascular events, showed that low handgrip strength accurately predicted cardiovascular events, ${ }^{11)}$ with this being the first study to clarify handgrip strength and CAVI as predictors of MACE.

Sarcopenia is defined by muscle mass, muscle strength, and walking speed. In this study, we used the diagnostic criteria developed by consensus report of the Asian Working Group for Sarcopenia. ${ }^{4}$ We used the SMI, muscle strength, and walking speed to diagnose sarcopenia. In this study, the SMI differed between the two groups, but it was not an independent factor in regression analysis. By contrast, handgrip strength was an independent factor and is therefore considered to be an index that correlates with prognosis. With regard to other analyzed variables, diabetes laboratory data, medication history, and coronary risk factors such as hypertension and dyslipidemia were not independent predictive factors in regression analysis. Hypercholesterolemia and hypertension are known risk factors for coronary diseases in patients with DM. In addition, the use of statins is known to reduce the risk of macrovascular events, ${ }^{21)}$ and ACE inhibitors and calcium channel blockers can significantly reduce the risk of stroke. Furthermore, the risk of coronary artery disease is known to be suppressed by calcium channel blockers. ${ }^{22)}$ The patients in this study were hospitalized for control of DM and were discharged with instructions regarding exercise, drug therapy, and dietary measures to address coronary artery disease. Therefore, it is possible that traditional coronary risk factors were not identified as independent factors in regression analysis in this study. Despite the background of our participants, however, handgrip strength was identified as an independent factor in regression analysis, so it may become important as a new prognostic factor.

Additional studies will be required to investigate laboratory data other than those examined here, as in patients with DM it is necessary to consider factors that affect handgrip strength and cardiovascular events, but the power of this study was insufficient to do so.

While in past studies, impaired endothelial nitric oxide synthesis played a role in the association between albuminuria and cardiovascular disease risk, in this study, while the duration of DM was similar in the two groups, the sarcopenia group demonstrated a significantly higher degree of albuminuria and significantly reduced FMD compared to the non-sarcopenia group. Furthermore, there are indications that endothelial dysfunction and chronic inflammation, both of which play an important role in the development of atherothrombosis, are present in the early stages of renal insufficiency. ${ }^{23-26)}$ In what might be associ- ated with cardiovascular events in patients with DM and sarcopenia because endothelial function is known to correlate with albuminuria, and while this study did not measure inflammatory factors, deterioration of endothelial function was observed in the sarcopenia group. Although it is unknown whether sarcopenia is the cause of endothelial dysfunction, handgrip strength is thought to be associated with endothelial dysfunction and may be associated with prognosis for this reason. In order to clarify the cause of sarcopenia cardiovascular events in patients with $\mathrm{DM}$, further research is needed to clarify the etiology of sarcopenia.

In this study, handgrip strength was the most relevant predictor of MACE. Although this study was able to suggest a relationship between handgrip strength and MACE, various factors are thought to be related, and further research is needed on each relationship.

Limitations: First, our study is conducted at one institution, and so is not a multi-center study. Also it has a small number of diabetic patients. Second, in this study, diabetic patients were treated only with conventional diabetes, without intervention with drugs or the like. Third, this study is conducted on patients hospitalized for diabetes control, targeting patients with poor diabetes control at admission. The fourth limitation is that the treadmill test has low sensitivity and specificity for coronary artery disease. Since this study recognizes the above limitations, further research is needed to resolve these limitations.

\section{Conclusions}

In what suggests that the handgrip strength test might be useful in the management of patients with DM who are at high risk of cardiovascular outcomes, handgrip strength is an indicator of sarcopenia in diabetic patients, and like CAVI, it was found to be independently associated with the prevalence of MACE.

\section{Disclosure}

Conflicts of interest: No COI to disclose.

IRB information: The Ethics Committee of Nara City Hospital (approval number 36)

\section{References}

1. Rosenberg IH. Summary comments. Am J Clin Nutr 1989; 50: 1231-3.

2. Rosenberg IH. Sarcopenia: origins and clinical relevance. J Nutr 1997; 127 Supplement: 990S-991S.

3. Cruz-Jentoft AJ, Baeyens JP, Bauer JM, et al. European Working Group on Sarcopenia in Older People. Sarcopenia: European consensus on definition and diagnosis: report of the European Working Group on Sarcopenia in Older People. Age Ageing 2010; 39: 412-23.

4. Chen LK, Liu LK, Woo J, et al. Sarcopenia in Asia: consensus report of the Asian Working Group for Sarcopenia. J Am Med Dir Assoc 2014; 15: 95-101.

5. Goodpaster BH, Carlson CL, Visser M, et al. Attenuation of skeletal muscle and strength in the elderly: the Health $\mathrm{ABC}$ Study. J Appl Physiol 2001; 90: 2157-65.

6. Huxley R, Barzi F, Woodward M. Excess risk of fatal coronary 
heart disease associated with diabetes in men and women: metaanalysis of 37 prospective cohort studies. BMJ 2006 Jan 14 332: 73-8.

7. Tajiri Y, Kato T, Nakayama H, Yamada K. Reduction of skeletal muscle, especially in lower limbs, in Japanese type 2 diabetic patients with insulin resistance and cardiovascular risk factors. Metab Syndr Relat Disord 2010; 8: 137-42.

8. Park SW, Goodpaster BH, Strotmeyer ES, et al. Decreased muscle strength and quality in older adults with type 2 diabetes: the health, aging, and body composition study. Diabetes 2006; 55: 1813-8.

9. Kim JH, Cho JJ, Park YS. Relationship between sarcopenic obesity and cardiovascular disease risk as estimated by the Framingham risk score. J Korean Med Sci 2015; 30: 264-71.

10. Onoue Y, Izumiya $\mathrm{Y}$, Hanatani $\mathrm{S}$, et al. A simple sarcopenia screening test predicts future adverse events in patients with heart failure. Int J Cardiol 2016; 215: 301-6.

11. Hamasaki H, Kawashima Y, Katsuyama H, Sako A, Goto A, Yanai H. Association of handgrip strength with hospitalization, cardiovascular events, and mortality in Japanese patients with type 2 diabetes. Sci. Rep. 2017; 7: 7041.

12. Baumgartner RN, Koehler KM, Gallagher D, et al. Epidemiology of sarcopenia among the elderly in New Mexico. Am J Epidemiol 1998; 147: 755-63.

13. Corretti MC, Anderson TJ, Benjamin EJ, et al. Internationa Brachial Artery Reactivity Task Force. Guidelines for the ultrasound assessment of endothelial-dependent flow-mediated vasodilation of the brachial artery: a report of the International Brachial Artery Reactivity Task Force. J Am Coll Cardiol 2002; 39 257-65.

14. Thijssen DH, Black MA, Pyke KE, et al. Assessment of flowmediated dilation in humans: a methodological and physiological guideline. Am J Physiol Heart Circ Physiol 2011; 300: H2H12.

15. Shirai K, Utino J, Otsuka K, Takata M. A novel blood pressureindependent arterial wall stiffness parameter; cardio-ankle vascular index (CAVI). J Atheroscler Thromb 2006 Apr; 13: 101-7.

16. Atkins JL, Whincup PH, Morris RW, Lennon LT, Papacosta O, Wannamethee SG. Sarcopenic obesity and risk of cardiovascular disease and mortality: a population-based cohort study of older men. J Am Geriatr Soc 2014 Feb; 62: 253-60

17. Lee YH, Kim JS, Jung SW, et al. Gait speed and handgrip strength as predictors of all-cause mortality and cardiovascular events in hemodialysis patients. BMC Nephrol 2020 May 6; 21: 166.

18. Artero EG, Lee DC, Ruiz JR, et al. A prospective study of muscular strength and all-cause mortality in men with hypertension. J Am Coll Cardiol 2011 May 3; 57: 1831-7.

19. Stenholm S, Sallinen J, Koster A, et al. Association between obesity history and hand grip strength in older adults--exploring the roles of inflammation and insulin resistance as mediating factors. J Gerontol A Biol Sci Med Sci 2011 Mar; 66: 341-8.

20. Leong DP, Teo KK, Rangarajan S, et al. Prognostic value of grip strength: findings from the Prospective Urban Rural Epidemiology (PURE) study. Lancet 2015; 386: 266-73.

21. Cholesterol Treatment Trialists' (CTT) Collaborators, Kearney PM, Blackwell L, et al. Efficacy of cholesterol-lowering therapy in 18,686 people with diabetes in 14 randomised trials of statins: a meta-analysis. Lancet 2008 Jan 12; 371: 117-25.

22. Turnbull F, Neal B, Algert C, et al. Effects of different blood pressure-lowering regimens on major cardiovascular events in individuals with and without diabetes mellitus: results of prospectively designed overviews of randomized trials. Arch Intern Med 2005 Jun 27; 165: 1410-9.

23. Stehouwer CD, Henry RM, Dekker JM, Nijpels G, Heine RJ, Bouter LM. Microalbuminuria is associated with impaired brachial artery, flow-mediated vasodilation in elderly individuals without and with diabetes: further evidence for a link between microalbuminuria and endothelial dysfunction--the Hoorn Study. Kidney Int Suppl 2004; 92: S42-4.

24. De Jong PE, Curhan GC. Screening, monitoring, and treatment of albuminuria: public health perspectives. J Am Soc Nephrol 2006; 17: 2120-6.

25. Stam F, van Guldener C, Becker A, et al. Endothelial dysfunction contributes to renal function-associated cardiovascular mortality in a population with mild renal insufficiency: the Hoorn study. J Am Soc Nephrol 2006; 17: 537-45.

26. Miura M, Sakata Y, Miyata S, et al. Prognostic impact of subclinical microalbuminuria in patients with chronic heart failure. Circ J 2014; 78: 2890-8. 